\title{
Results from a Test-to-Release from Isolation Strategy Among Fully Vaccinated National Football League Players and Staff Members with COVID-19 - United States, December 14-19, 2021
}

\author{
Christina D. Mack, $\mathrm{PhD}^{1}$; Erin B. Wasserman, $\mathrm{PhD}^{1}$; Marie E. Killerby, VetMB ${ }^{2}$; Rieza H. Soelaeman, $\mathrm{PhD}^{2}$; Aron J. Hall, DVM²; \\ Adam MacNeil, $\mathrm{PhD}^{2}$; Deverick J. Anderson, MD³; Patti Walton, MHSA ${ }^{4}$; Saamir Pasha, MPH ${ }^{1}$; Emily Myers ${ }^{5}$; Catherine S. O’Neal, MD ${ }^{6}$; \\ Christopher J. Hostler MD ${ }^{3}$; Navdeep Singh, $\mathrm{MD}^{7}$; Thom Mayer, $\mathrm{MD}^{8}$, Allen Sills, $\mathrm{MD}^{5}$
}

During December 2021, the United States experienced a surge in COVID-19 cases, coinciding with predominance of the SARS-CoV-2 B.1.1.529 (Omicron) variant (1). During this surge, the National Football League (NFL) and NFL Players Association (NFLPA) adjusted their protocols for test-to-release from COVID-19 isolation on December 16, 2021, based on analytic assessments of their 2021 test-to-release data. Fully vaccinated* persons with COVID-19 were permitted to return to work once they were asymptomatic or fever-free and experiencing improving symptoms for $\geq 24$ hours, and after two negative or high cycle-threshold $(\mathrm{Ct})$ results $(\mathrm{Ct} \geq 35)$ from either of two reverse transcription-polymerase chain reaction (RT-PCR) tests $^{\dagger}(2)$. This report describes data from NFL's SARS-CoV-2 testing program (3) and time to first negative or $\mathrm{Ct} \geq 35$ result based on serial COVID-19 patient testing during isolation. Among this occupational cohort of 173 fully vaccinated adults with confirmed COVID-19 during December 14-19, 2021, a period of Omicron variant predominance, $46 \%$ received negative test results or had a subsequent RT-PCR test result with a $\mathrm{Ct} \geq 35$ by day 6 postdiagnosis (i.e., concluding 5 days of isolation) and $84 \%$ before day 10 . The proportion of persons with positive test results decreased with time, with approximately one half receiving positive RT-PCR test results after postdiagnosis day 5 . Although this test result does not necessarily mean these persons are infectious (RT-PCR tests might continue to return positive results long after an initial positive result) (4), these findings indicate that persons with

\footnotetext{
*Fully vaccinated was defined as $\geq 14$ days after primary vaccination series completion, with or without a booster. Partially vaccinated was defined by NFL as receipt of 1 dose of a 2-dose series or $<14$ days after primary vaccine series completion. Under NFL-NFLPA protocols, persons with 1 dose of a 2-dose mRNA vaccination series and a documented history of COVID-19 were subject to the same requirements as were fully vaccinated persons in the NFL-NFLPA protocols; however, these persons were excluded from analyses summarizing the test-to-release from isolation strategy.

${ }^{\dagger}$ SARS-CoV-2 tests included either laboratory-based real-time RT-PCR (BioReference Laboratories RT-PCR assay [Roche Cobas assay, Roche]) or point of care (POC) RT-PCR (Mesa Biotech Accula SARS-CoV-2 test, Mesa Biotech Inc.). The Roche Cobas RT-PCR test used received Food and Drug Administration (FDA) Emergency Use Authorization as a qualitative diagnostic assay with a Ct cutoff of $>40$. Use of this assay with alternative Ct cutoffs is not authorized by FDA. The Mesa Biotech Accula test produces a qualitative result (positive/negative) without Ct values.
}

COVID-19 should continue taking precautions, including correct and consistent mask use, for a full 10 days after symptom onset or initial positive test result if they are asymptomatic.

The NFL consists of 32 member clubs based in 24 states. NFL required COVID-19 vaccination (as of June 7, 2021) and boosters (as of December 27) for staff members who directly interact with players and incentivized but did not require vaccination among players. By October 13, 2021, the NFL population vaccination rate was $>96 \%$ (staff members $>99 \%$; players >94\%) (5). For most of the 2021 season, fully vaccinated persons were tested weekly and unvaccinated persons were tested daily by RT-PCR; antigen testing was not used. On December 18, 2021, testing protocols transitioned to symptom- or exposure-based testing, determined through enhanced symptom screening and contact tracing for fully vaccinated persons, resulting in approximately two thirds the number of tests, but retaining a high level of case detection (G). Around the same time, NFL also updated test-to-release protocols (Box). Daily testing was requested for all fully vaccinated persons with COVID-19, who could be released from isolation if they were asymptomatic or fever-free with symptoms improved for 24 hours with medical staff clearance, and receipt of two negative or $\mathrm{Ct} \geq 35 \mathrm{RT}-\mathrm{PCR}$ test results (point of care [POC] or laboratory-based) within 24 hours (2). Although $\mathrm{Ct}$ values have not been validated as a measure of infectiousness (7), a conservative Ct cutoff was chosen by NFL based on higher $\mathrm{Ct}$ values correlating with limited culturable virus in other studies (8).

COVID-19 cases $^{\S}$ identified during December 12 , 2021-January 1, 2022, were stratified by whether persons were fully vaccinated, partially vaccinated, or unvaccinated. SARS-CoV-2 tests included either laboratory-based real-time RT-PCR (BioReference Laboratories RT-PCR assay [Roche Cobas assay], Roche) or POC RT-PCR (Mesa Biotech Accula SARS-CoV-2 Test, Mesa Biotech Inc.); both tests demonstrated

\footnotetext{
${ }^{\$}$ A COVID-19 case was defined as detection of SARS-CoV-2 RNA using a diagnostic nucleic acid amplification test performed by a Clinical Laboratory Improvement Amendments-certified provider and confirmed by additional RT-PCR tests alongside clinical adjudication. For COVID-19 cases, day of diagnosis ("day zero") was defined as the day of first positive test result, which in this setting of high access to testing was often the day of symptom onset.
} 


\section{June 16, 2021 (NFL season start)}

- Requested daily laboratory-based and POC RT-PCR testing* for all fully vaccinated ${ }^{\dagger}$ persons with COVID-19

- Permitted fully vaccinated persons with COVID-19 to voluntarily return to work early (before 10 days since first positive test) upon meeting the following testing and clinical criteria:

- Testing criteria

- Two negative laboratory-based RT-PCR tests separated by $\geq 24$ hours

- One negative POC RT-PCR test before entry on the date of entry

- Clinical criteria

- Asymptomatic for 48 hours

- Medical clearance by the team physician

- Review of test results by the NFL Chief Medical Officer and clinical consultants

- Upon return, all persons followed existing NFL-NFLPA masking and behavioral protocols

\section{November 9, 2021}

- Requested daily laboratory-based RT-PCR testing for all fully vaccinated persons with COVID-19

- Permitted fully vaccinated persons with COVID-19 to voluntarily return to work early (before 10 days since first positive test) upon meeting the following testing and clinical criteria

- Testing criteria

- Days 1-6 after a confirmed positive test result: two negative laboratory-based RT-PCR test results separated by $\geq 24$ hours

- Days 7-10 after a confirmed positive test result: 1) one negative laboratory-based RT-PCR test result; and 2) one negative POC RT-PCR test result before entry on the day following specimen collection of initial laboratory-based RT-PCR test (taken $\geq 24$ hours later)

- Clinical criteria: Days 1-6 or Days 7-10

- Asymptomatic for 48 hours (other than loss of taste or smell)

- Medical clearance by the team physician

- Confirmation of test results by the NFL Chief Medical Officer and clinical consultants

- Upon return, all persons followed existing NFL-NFLPA masking and behavioral protocols

See box footnotes on the next page.

previous high performance in this setting (3) (NFL, unpublished data). Sequence analysis was performed using Illumina COVIDSeq (9) for a portion of cases. Symptoms throughout illness were clinically monitored but not centrally recorded; NFL clubs requesting early return permission were required to provide documentation of asymptomatic or improving symptom status. Clubs were requested to closely monitor persons returning and report any new symptoms; persons returning were required to adhere to applicable NFL-NFLPA masking policies.

This report describes results from the NFL test-to-release from isolation strategy for fully vaccinated persons with confirmed COVID-19 during December 14-19, 2021. These dates reflect a period during which the Omicron variant accounted for $97 \%$ of sequenced viruses, and protocols requested COVID-19 patients test daily for 10 days after diagnosis (isolation ended on day 5 beginning December 28, 2021). Fully vaccinated persons were included if they were tested $\geq 6$ times during infection, with a final test on day 8 or 9 postdiagnosis or achieved two negative or $\mathrm{Ct} \geq 35 \mathrm{RT}-\mathrm{PCR}$ test results (after which testing ceased). Subsequent transmission from persons returning from isolation early could not be ascertained because of high community COVID-19 incidence and limited Omicron genomic sequencing diversity. The analysis in this report was conducted by IQVIA on behalf of NFL with CDC's support. This activity was reviewed by CDC and was conducted consistent with applicable federal law and CDC policy. 9

During December 12, 2021-January 1, 2022, a surge in COVID-19 cases occurred among NFL players and staff, with an average of 336 cases per week, compared with 30 cases per week during the preceding 3 months. Sixty-six (53\%) cases occurred among 125 unvaccinated persons, three (23\%)

\footnotetext{
95 C.F.R. part 46, 21 C.F.R. part 56; 42 U.S.C. Sect. 241(d); 5 U.S.C. Sect. 552a; 44 U.S.C. Sect. 3501 et seq.
} 


\section{December 16, $2021^{\S}$}

- Strongly requested daily testing using laboratory-based and POC RT-PCR after a confirmed positive test result for all fully vaccinated persons with COVID-19

- Permitted fully vaccinated persons with COVID-19 to voluntarily return to work early (before 10 days since first positive test result) upon meeting the following testing and clinical criteria

- Testing criteria: two samples collected $\leq 24$ hours of each other on Day 1 or thereafter (may be collected on the same day; must be separate swabs)

- Two negative or $\mathrm{Ct} \geq 35$ laboratory-based RT-PCR test results; or

- One negative or $\mathrm{Ct} \geq 35$ laboratory-based RT-PCR test result and one negative POC RT-PCR test result; or

- Two negative POC RT-PCR test results

- Clinical criteria

- Asymptomatic or fever-free and improving symptoms for $\geq 24$ hours

- Medical clearance by the team physician

- Confirmation of test results by the NFL Chief Medical Officer and clinical consultants

- Although Ct values are not a measure of infectiousness a Ct cutoff of 35 was chosen based on higher $\mathrm{Ct}$ values correlating with limited culturable virus using alternative RT-PCR tests**

- Upon return, all persons followed existing NFL-NFLPA masking and behavioral protocols

\section{December 28, 2021}

- Allowed for day 5 return if fever-free for $\geq 24$ hours, asymptomatic or improving symptoms, and cleared by team physician, regardless of vaccination status ${ }^{\dagger \dagger}$

- Once cleared from isolation (between Days 5 and 10), all persons were required to wear a KN95 or N95 mask at all times other than when actively engaged in physical activity at practice or in a game until 10 days following positive test result, at which time they returned to existing NFL-NFLPA masking protocols

- Fully vaccinated persons with COVID-19 could return before Day 5 by meeting the December 16 protocol criteria. Upon return, persons were required to follow existing NFL-NFLPA masking and behavioral protocols.

Abbreviations: FDA = Food and Drug Administration; NFL = National Football League; NFLPA = National Football League Players Association; POC = pointof-care; RT-PCR = reverse transcription-polymerase chain reaction.

* SARS-CoV-2 tests included either laboratory-based real-time RT-PCR (BioReference Laboratories RT-PCR assay [Roche Cobas assay], Roche) or POC RT-PCR (Mesa Biotech Accula SARS-CoV-2 test, Mesa Biotech Inc.); the Roche Cobas RT-PCR test used received FDA Emergency Use Authorization as a qualitative diagnostic assay with a $\mathrm{Ct}$ cutoff of $>40$. Use of this assay with alternative Ct cutoffs is not authorized by FDA. The Mesa Biotech Accula test produces only a qualitative result (positive/negative) and no Ct values.

$\dagger$ Fully vaccinated was defined as $\geq 14$ days after primary vaccine series completion, with or without a booster. Under NFL-NFLPA protocols, persons with 1 dose of a 2-dose mRNA vaccination series and a documented history of COVID-19 were subject to the same requirements as fully vaccinated persons in the NFLNFLPA protocols; however, these persons were excluded from analyses summarizing the test-to-release from isolation strategy.

$\$_{\mathrm{https}} / /$ www.nfl.com/news/nfl-updates-covid-19-protocols-to-allow-for-quicker-return-from-quarantine-for-v

I https://www.cdc.gov/coronavirus/2019-ncov/lab/faqs.html

** https://academic.oup.com/cid/article/73/11/e3884/6018217

${ }^{\dagger \dagger}$ https://www.cdc.gov/media/releases/2021/s1227-isolation-quarantine-guidance.html

among 13 partially vaccinated persons, and $924(14 \%)$ among 6,443 fully vaccinated persons. ${ }^{* *}$ Among 117 (12\%) cases with a sequenced virus isolate during this period, $111(95 \%)$ were classified as Omicron and six (5\%) as the SARS-CoV-2 B.1.617.2 (Delta) variant.

\footnotetext{
** The remaining 15 cases occurred among 75 persons with 1 dose of a 2-dose mRNA vaccination series and a documented history of COVID-19, who were subject to the same requirements as fully vaccinated persons in the NFLNFLPA protocols. These persons were not included in analyses summarizing the test-to-release from isolation strategy.
}

Using data from December 14-19, 2021, a total of $218(5 \%)$ cases were identified in NFL players and staff members among 4,134 persons tested; 87 (97\%) of 90 sequenced isolates were Omicron. Among 201 COVID-19 cases in fully vaccinated persons that were either confirmed as Omicron or unsequenced (presumed Omicron), 173 (86\%) tested for isolation release; the remaining $28(14 \%)$ did not test frequently enough for inclusion. Among these 173 persons, 53 (31\%) obtained at least one negative or Ct $\geq 35$ RT-PCR test result on or before day 5 postdiagnosis, 79 (46\%) on or before day 6 (concluding 5 full days of isolation), and 146 (84\%) within 10 days after 
diagnosis. Among the 146 persons with at least one negative or $\mathrm{C} t \geq 35$ RT-PCR test result in the 10 days after diagnosis, the median interval from diagnosis to first negative result was 6 days (IQR $=5-8$ days); among all 173 persons, the median interval was 7 days (IQR $=5-9$ days) (Figure 1 ).

Overall, 130 (75\%) of 173 persons tested for return from isolation met NFL-NFLPA protocol testing criteria before 10 days, with median interval to second negative or $\mathrm{Ct} \geq 35$ test result of 7 days (IQR $=5-8$ days); $122(71 \%)$ met both testing and clinical criteria and were eligible to return to work before 10 days after diagnosis (Figure 2). Among the 130 persons who tested for early return from isolation, 116 applied for early return; the remaining 14 did not because either their job function did not require early return (six), or they were still symptomatic (six); two did not apply for early return for unknown reasons. Among 173 persons who tested for release from isolation, the median time to return to work was 8 days (IQR = 6-10 days). None of the persons who returned to work reported onset of new symptoms after early return during the 10 days after diagnosis.

\section{Summary \\ What is already known about this topic?}

On December 16, 2021, the National Football League (NFL) updated its test-to-release from COVID-19 isolation protocols in response to increasing COVID-19 cases and predominance of the SARS-CoV-2 Omicron variant.

What is added by this report?

Among 173 vaccinated adults with COVID-19 undergoing serial reverse transcription-polymerase chain reaction (RT-PCR) testing during Omicron predominance, $46 \%$ received a negative or high cycle threshold RT-PCR test result on or before day 6 postdiagnosis.

What are the implications for public health practice? Although a positive RT-PCR test result does not necessarily indicate infectiousness, these data indicate that persons with COVID-19 should continue to take precautions, including correct and consistent mask use, for a full 10 days after symptom onset or after initial positive test result if they are asymptomatic.

FIGURE 1. Percentage of 173 fully vaccinated* COVID-19 patients (SARS-CoV-2 B.1.1.529 [Omicron] and unsequenced ${ }^{\dagger}$ ) with a negative or cycle-threshold $\geq 35^{\S}$ reverse transcription-polymerase chain reaction test result, by number of days after diagnosis - National Football League, United States, December 14-19, 2021

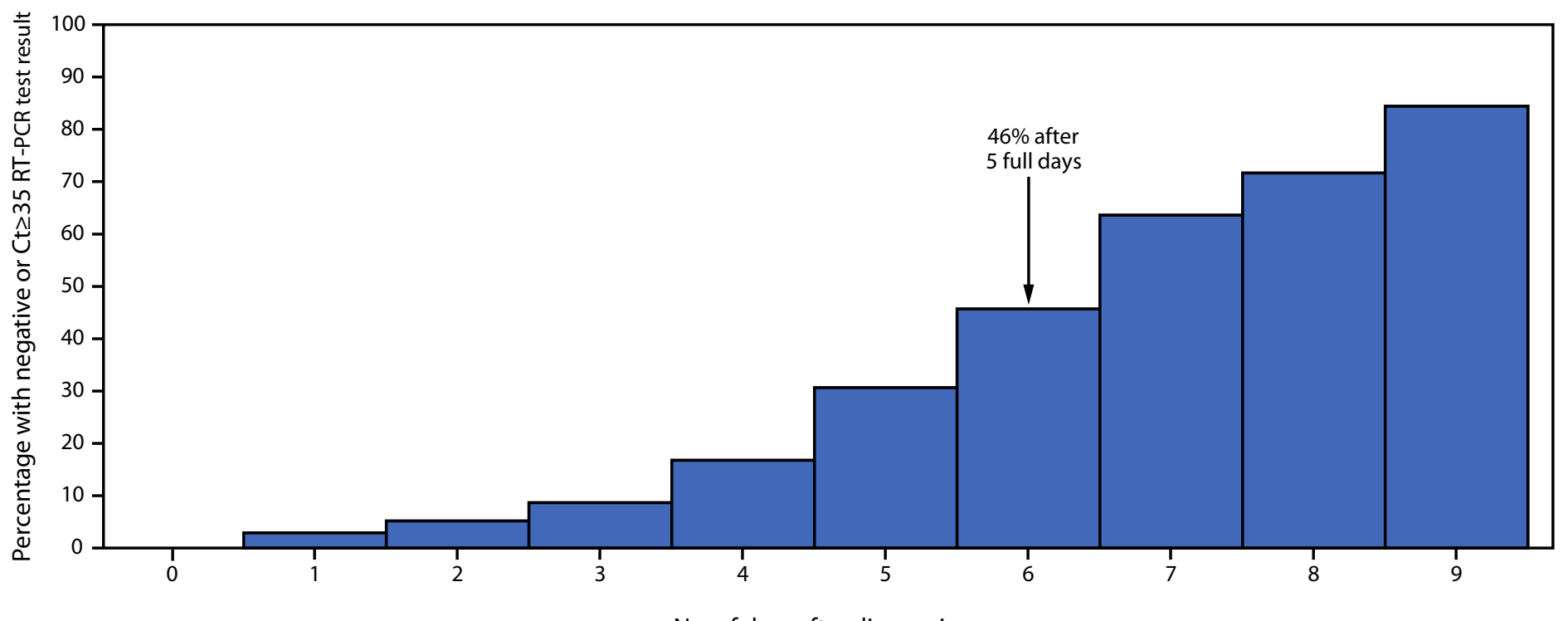

No. of days after diagnosis

Abbreviations: $\mathrm{Ct}=$ cycle threshold; $\mathrm{NFL}=$ National Football League; NFLPA = National Football League Players Association; $\mathrm{RT}-\mathrm{PCR}=$ reverse transcription-polymerase chain reaction.

* Fully vaccinated was defined as $\geq 14$ days after primary vaccine series completion. Under NFL-NFLPA protocols, persons with 1 dose of a 2-dose mRNA vaccination series and a documented history of COVID-19 were subject to the same requirements as fully vaccinated persons in the NFL-NFLPA protocols; however, these persons were excluded from analyses summarizing the test-to-release from isolation strategy.

† Among 173 cases, 77 (45\%) were sequenced as Omicron, and 96 (55\%) were not sequenced (unsequenced). During December 14-19, 2021, 87 (97\%) of 90 sequenced isolates from 218 total cases were Omicron, therefore unsequenced cases were presumed Omicron.

$\S$ Although Ct values are not a measure of infectiousness (https://www.cdc.gov/coronavirus/2019-ncov/lab/faqs.html) a Ct cutoff of 35 was chosen by NFL based on higher Ct values correlating with limited culturable virus using alternative RT-PCR tests (https://academic.oup.com/cid/article/73/11/e3884/6018217). 


\section{Discussion}

This report describes results from the NFL test-to-release from isolation strategy for fully vaccinated NFL players and staff members with COVID-19 during December 14-19, 2021, after emergence of the Omicron variant. Before day 10 postdiagnosis, $\geq 71 \%$ of infected persons were asymptomatic or significantly improved for 24 hours and had received two negative or $\mathrm{Ct} \geq 35$ test results and were therefore eligible to return to work under NFL-NFPLA protocols. Although persons were not systematically tested after return from isolation, and transmission after early return from isolation could not be documented, none of the persons who returned to work experienced new symptoms detected by in-facility symptom monitoring.

CDC recommends an additional 5 days of mask use when around others following an isolation period of 5 days, which can end when a person has been fever-free for 24 hours and other symptoms have improved (10). The results from this analysis support this masking recommendation, although infectiousness or transmission could not be assessed. Under

FIGURE 2. Test-to-release from isolation results among 201 fully vaccinated* COVID-19 patients (SARS-CoV-2 B.1.1.529 [Omicron] and unsequenced $^{\dagger}$ ) undergoing serial reverse transcription-polymerase chain reaction testing ${ }^{\S}$ to allow release from isolation - National Football League, December 14-19, $2021^{\uparrow, * *,+\dagger}$

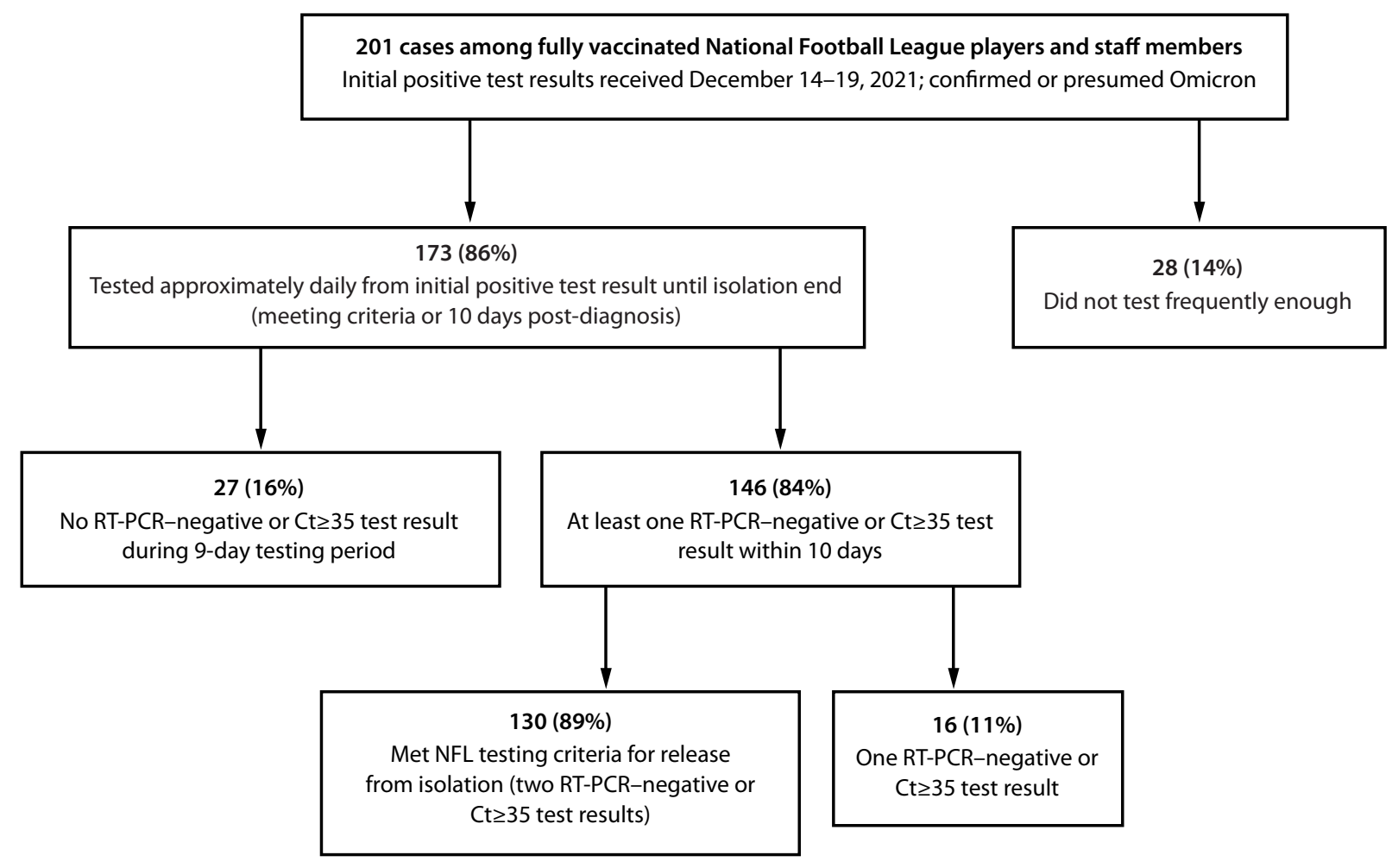

Abbreviations: $\mathrm{Ct}=$ cycle threshold; NFL $=$ National Football League; NFLPA = National Football League Players Association; POC $=$ point of care; RT-PCR $=$ reverse transcription-polymerase chain reaction.

* Fully vaccinated was defined as $\geq 14$ days after primary vaccine series completion, with or without a booster. Under NFL-NFLPA protocols, persons with 1 dose of a 2-dose mRNA vaccination series and a documented history of COVID-19 were subject to the same requirements as fully vaccinated persons in the NFL-NFLPA protocols; however, these persons were excluded from analyses summarizing the test-to-release from isolation strategy.

† Among 201 cases, 79 (39\%) were sequenced as Omicron, and 122 (61\%) were not sequenced (unsequenced). During December 14-19, 2021, 87 (97\%) of 90 sequenced isolates from 218 total cases were Omicron, therefore unsequenced cases were presumed Omicron.

$\S$ RT-PCR tests included either laboratory-based real-time RT-PCR (Bioreference Labs RT-PCR assay [Roche Cobas assay], Roche) or POC RT-PCR (Mesa Biotech Accula SARS-CoV-2 Test, Mesa Biotech Inc.).

" Among those who did not test frequently enough, 26 (93\%) had a job function that did not require regular attendance in facility or immediate return or were travel-related.

** Among 130 persons who met testing criteria, 122 (94\%) met testing and clinical criteria for release from isolation, six had continuing symptoms, and two did not apply for early return for unknown reasons.

${ }^{+\dagger}$ Although Ct values are not a measure of infectiousness (https://www.cdc.gov/coronavirus/2019-ncov/lab/faqs.html) a Ct cutoff of 35 was chosen by NFL based on higher Ct values correlating with limited culturable virus using alternative RT-PCR tests (https://academic.oup.com/cid/article/73/11/e3884/6018217). 
NFL's test-to-release from isolation strategy, approximately one half of fully vaccinated persons with COVID-19 had negative or $\mathrm{Ct} \geq 35 \mathrm{RT}-\mathrm{PCR}$ test results after 5 full days of isolation (i.e., on day 6 ), and $84 \%$ by day 10 . CDC advises that persons with access to tests might use an antigen test toward the end of the 5-day isolation period (10), because RT-PCR tests can continue to return positive results with high $\mathrm{Ct}$ values intermittently after an initial positive result (4). Ct values are not a validated measure of infectiousness; however, the data from this study indicate that persons can receive a positive test result after a 5-day isolation period. It is therefore important for persons with COVID-19 to continue to wear masks correctly and consistently for a full 10 days after symptom onset or after an initial positive test result if they are asymptomatic (10).

The findings in this report are subject to at least six limitations. First, these data are based on results from highly sensitive RT-PCR tests, whereas CDC advises that persons with access to rapid antigen tests might use these toward the end of the 5-day isolation period (10). Second, because of high community incidence of COVID-19 during this period and limited Omicron genomic sequencing diversity, subsequent transmission postisolation release cannot be ascertained. Third, this investigation is limited to a population of predominantly adult men in an occupational setting, who are likely healthier than the general population, and the NFL testing program differed from U.S. community-based testing; thus, these findings might not be generalizable to other populations. Fourth, Ct values from RT-PCR tests do not necessarily indicate viral load or infectiousness in an individual person, and other factors, including specimen collection and handling, can affect $\mathrm{Ct}$ values (7). The use of a $\mathrm{Ct} \geq 35$ cutoff-point for these RT-PCR tests did not have Emergency Use Authorization and was not systematically evaluated nor had either assay been evaluated against the Omicron variant. Fifth, because of rapid administration of booster vaccine doses during the investigation period, cases could not be reported by booster dose receipt status. Finally, symptom data were not available throughout illness; reporting of asymptomatic status on negative test receipt was based on NFL clubs' submission for early return permission.

In this report describing test-to-release from isolation strategies in an occupational sport setting and RT-PCR test results among fully vaccinated persons with Omicron variant COVID-19, approximately one half had a negative result or a $\mathrm{Ct}$ value $\geq 35$ on or before day 6 , concluding 5 days of isolation. Although a positive RT-PCR test result does not necessarily indicate infectiousness, these data indicate that persons with COVID-19 should continue taking precautions, including correct and consistent mask use, for a full 10 days after symptom onset or after initial positive test result if they are asymptomatic.

\section{Acknowledgments}

Monita Patel, CDC; National Football League (NFL) club infection control officers; NFL team athletic trainers, physicians, and orthopedists; NFL Players Association; NFL COVID-19 Advisory and Operational Team including Dawn Aponte, NFL; Michele Best, University of Maryland Capital Region Health; Meghan C. Carroll, NFL; M. Anthony Casolaro, Washington Football Team; Molly Delaney, NFL; Daniel Eichner, Sports Medicine Research and Testing Laboratory; Jacob Frank, NFL; John Lynch, Department of Medicine, University of Washington; Jimmie Mancell, Department of Medicine, University of Tennessee Health Science Center; Damion Martins, Atlantic Sports Health, Morristown Medical Center; John Mellody, NFL; Eric Sugarman, Minnesota Vikings; IQVIA and NFL contact tracing and technical team including Megan Miraval, Ally Thrasher, Sabrina Trujillo Smith, Madeline Lassiter, Emily Watson, Kristin Archibald, Emma Bergqvist, Paul Blalock, Justin Reid, Julie Griffith, Kristina Zeidler; Sequencing partners at Tempus and Yale School of Public Health.

Corresponding author: Christina D. Mack, Christina.Mack@iqvia.com.

\begin{abstract}
${ }^{1}$ IQVIA Real-World Solutions, Durham, North Carolina; ${ }^{2}$ CDC COVID-19 Emergency Response Team; ${ }^{3}$ Infection Control Education for Major Sports, LLC, Chapel Hill, North Carolina; ${ }^{4}$ Williamson Medical Center, Franklin, Tennessee; ${ }^{5}$ National Football League, New York, New York; ${ }^{6}$ Louisiana State University Health Sciences Center, Baton Rouge, Louisiana; ${ }^{7}$ Eden Medical Center, Castro Valley, California; ${ }^{8}$ National Football League Players Association, Washington, D.C.
\end{abstract}

All authors have completed and submitted the International Committee of Medical Journal Editors form for disclosure of potential conflicts of interest. Christina D. Mack, Erin B. Wasserman, and Saamir Pasha report full-time employment by IQVIA. Deverick J. Anderson reports receipt of royalties for authorship from UpToDate Online, LLC and co-ownership of Infection Control Education for Major Sports, LLC. Patti Walton reports consulting fees from NFL, honoraria for presentation from Ortho Clinical Diagnostics, payment as an expert witness regarding laboratory practices, support for travel to meetings from the American Society for Clinical Pathology (ASCP), serving on the Board of Directors of the Clinical Laboratory Management Association (2019-2021), membership on the ASCP Board of Governors (2019-current), and Chairmanship of the Tennessee Medical Laboratory Licensing Board (2019-2021). Catherine O'Neal reports consulting fees from NFL. Christopher J. Hostler reports co-ownership of Infection Control Education for Major Sports, LLC. Allen Sills and Emily Myers report employment by NFL. Navdeep Singh reports receipt of consulting fees from NFL and the Las Vegas Raiders. No other potential conflicts of interest were disclosed. 


\section{References}

1. CDC. Variant proportions. Atlanta, GA: US Department of Health and Human Services, CDC; 2021. https://covid.cdc.gov/ covid-data-tracker/\#variant-proportions

2. National Football League. NFL updates COVID-19 protocols to allow for quicker return from quarantine for vaccinated individuals. New York, NY: National Football League, 2021. https:/www.nfl.com/news/ nfl-updates-covid-19-protocols-to-allow-for-quicker-return-fromquarantine-for-v

3. Mack CD, Osterholm M, Wasserman EB, et al. Optimizing SARS-CoV-2 surveillance in the United States: insights from the National Football League Occupational Health Program. Ann Intern Med 2021;174:1081-9. PMID:34125571 https://doi.org/10.7326/M21-0319

4. Mack CD, DiFiori JD, Tai C, et al. SARS-CoV-2 transmission risk among National Basketball Association players, staff, and vendors exposed to individuals with positive test results after COVID-19 recovery during the 2020 regular and postseason. JAMA Intern Med 2021;181(7):960-66. PMID:33885715 https://doi.org/10.1001/ jamainternmed.2021.2114

5. CDC. For NFL players, leadership and teamwork builds COVID-19 vaccine confidence. Atlanta, GA: US Department of Health and Human Services, CDC; November 12, 2021. https://www.cdc.gov/vaccines/ covid-19/health-departments/features/NFL.html
6. National Football League. New COVID-19 protocols to feature targeted testing of fully vaccinated players, staff. New York, NY: National Football League, 2021. https://www.nfl.com/news/new-covid-19-protocol-tofeature-targeted-testing-of-fully-vaccinated-players-st

7. CDC. Frequently asked questions about Coronavirus (COVID-19) for laboratories. Atlanta, GA: US Department of Health and Human Services, CDC; November 12, 2021. https://www.cdc.gov/ coronavirus/2019-ncov/lab/faqs.html

8. Jefferson T, Spencer EA, Brassey J, Heneghan C. Viral cultures for Coronavirus disease 2019 infectivity assessment: a systematic review. Clin Infect Dis 2021;73:e3884-99. PMID:33270107 https://doi. org $/ 10.1093 / \mathrm{cid} / \mathrm{ciaa} 1764$

9. Vogels CBF, Breban MI, Ott IM, et al. Brazil-UK CADDE Genomic Network; Network for Genomic Surveillance in South Africa. Multiplex qPCR discriminates variants of concern to enhance global surveillance of SARS-CoV-2. PLoS Biol 2021;19:e3001236. PMID:33961632 https://doi.org/10.1371/journal.pbio.3001236

10. CDC. Quarantine and isolation. Atlanta, GA: US Department of Health and Human Services, CDC; January 27, 2022. https://www.cdc.gov/ coronavirus/2019-ncov/your-health/quarantine-isolation.html 